\title{
O TRABALHO INFANTIL EM FOCO: ALGUMAS INTERLOCUÇÕES A PARTIR DOS ESTUDOS EM RESILIÊNCIA
}

\author{
Aline Madia Mantovani'; Anderson dos Santos Carvalho² \\ 1Universidade Estadual Paulista - UNESP, Programa de Pós Graduação em Educação, Presidente Prudente, SP. \\ ${ }^{2}$ Universidade Metodista de Piracicaba - UNIMEP, Mestrado em Educação Física, Piracicaba, SP. E-mail: \\ alinemadia@hotmail.com.
}

\section{RESUMO}

O presente estudo tem como objetivo compreender o trabalho infantil em suas interlocuções com os estudos em resiliência a partir da fala de adultos com histórico de trabalho infantil estudantes em turmas de Educação de Jovens em Adultos (EJA). Por meio da utilização de questionários e entrevistas semiestruturadas, em duas escolas públicas estaduais de Presidente Prudente/SP buscou-se investigar o envolvimento precoce em atividades laborais nas modalidades doméstico, rural e informal urbano, na tentativa de se entender os fatores de risco e de proteção que encaminharam esses participantes ao desenvolvimento de processos de resiliência. Ficaram claros os pontos negativos e positivos dessa inserção precoce em trabalho, assim como os fatores de risco e proteção advindos a partir dessas atividades, sendo a família, os amigos e a comunidade os recursos disponíveis e acessados por esses participantes.

Palavras-chave: Trabalho infantil. Resiliência. Adultos. Educação de Jovens e Adultos.

\section{THE CHILD LABOUR IN FOCUS: SOME INTERLOCUTIONS FROM STUDY ON RESILIENCE}

\begin{abstract}
This study aims to understand the child labor in its dialogues with the studies on resilience from the speech of adults with child labor history students at Educação de Jovens e Adultos (EJA) classes. Through the use of questionnaires and semi-structured interviews in two public schools in Presidente Prudente/SP sought to investigate early involvement in work activities in the domestic, rural and urban informal modalities, in an attempt to understand the risk factors and protection forwarded these participants to develop resilience processes. They became clear the negatives and positives of this early integration into work, as well as the risk factors and protection arising from these activities and family, friends and community resources available and accessed by those participants.
\end{abstract}

Keywords: Child labour. Resilience. Adults. Youth and Adult Education. 


\section{INTRODUÇÃO}

O trabalho infantil é um fenômeno complexo, envolto a inúmeros fatores de risco e possíveis resultados positivos, que se constrói de forma culturalmente embasada pelos sujeitos em seu contexto. Ao analisar os dados históricos e legais sobre o trabalho infantil no Brasil percebemos que se trata de uma categoria social fortemente ligada às mudanças no mundo do trabalho e aos aspectos sociais, econômicos e políticos presentes na vida da população (MONDINI, 2011).

Para Nunes (2009), após a Revolução Industrial em meados do século XVIII essa inserção da criança e do adolescente no mercado de trabalho passou a tomar grandes proporções e atingir um grande contingente de indivíduos. Ao lado do novo modelo econômico implantado (o Capitalismo) a Revolução expandiu-se pelo mundo, fazendo com que os trabalhadores, até então detentores de todo o processo produtivo, artesãos ou pequenos agricultores, perdessem o controle do mesmo e começassem a trabalhar para um patrão, dono das máquinas e do lucro adquirido.

Outro ponto a considerar é que a discussão da resiliência não deve se centrar apenas sobre os aspectos internos, as características pessoais e os atributos dos indivíduos para superarem as adversidades. Por essa perspectiva, o indivíduo é o único responsável pelo seu sucesso ou fracasso no processo de superação das adversidades, desconsiderando-se as dimensões mais culturais e os determinantes sociais, econômicos e históricos ligados às adversidades e que exigem maior investimento do Estado (LIBÓRIO, UNGAR, 2010).

Por essa via, estamos nos fundamentando em uma perspectiva cultural da resiliência, na tentativa de demonstrar que associar o trabalho infantil apenas a causas econômicas (como a pobreza, por exemplo) representa um erro com consequências graves para a efetivação e direcionamento de políticas públicas (que, na maioria das vezes, visam à erradicação do trabalho infantil sem uma análise cultural, subjetiva e contextual sobre sua ocorrência).

Em termos de definição, considera-se infantil toda e qualquer atividade que se executa antes da idade mínima prevista em lei (14 anos), inclusive quando esta inserção prejudicar e/ou inviabilizar a escolaridade, o lazer, o descanso, a saúde e a integridade de quem a exerce, configurando-se como uma atividade violadora de direitos fundamentais (BRASIL, 1990; LIBÓRIO, 2009; MANTOVANI, 2012).

A fim de melhor direcionar as políticas públicas e ações voltadas à erradicação do trabalho infantil, principalmente quando das suas piores formas (OIT, Convenção 182), convencionou-se classificar $a$ inserção em trabalho infantil em três modalidades: doméstico, informal urbano e rural. O trabalho infantil doméstico consiste nas atividades de lavar, passar, cozinhar, limpar e cuidar de bebês, idosos e animais domésticos, realizadas por crianças e/ou adolescentes por um período longo de tempo e por vários dias, sem a supervisão de um adulto e com/sem alguma forma de remuneração, diferentemente do trabalho doméstico configurado como 'ajuda', ou seja, as atividades esporádicas, que podem ter um adulto para auxiliar e algum tipo de remuneração (ALBERTO, NUNES, CAVALCANTE, SANTOS, 2005; LIBÓRIO, 2009).

Já o trabalho infantil informal urbano caracteriza-se pelo envolvimento de crianças e adolescentes em atividades inapropriadas para sua faixa etária, sem a regulamentação legal que garante direitos trabalhistas fundamentais (como descanso semanal renumerado e carteira de trabalho assinada) e que pode ser um fator de comprometimento da escolarização, da segurança e de atividades como lazer, cultura e desenvolvimento saudável a quem o exerce nas áreas urbanas (LIBÓRIO, 2009; MANTOVANI, 2012).

Por último, estão as atividades realizadas no meio rural - como arar a terra, cuidar de animais, cultivar o solo, dentre outras - tanto em fazendas comerciais como em áreas familiares, com longas horas de trabalho, em condições precárias e perigosas e com difícil acesso para instituições escolares (STROPASOLAS, 2012). 
Longe de defendermos o trabalho infantil, é possível observar em todas essas situações laborais que, apesar dos riscos, algumas pesquisas indicam que a percepção das crianças e adolescentes quanto ao trabalho desenvolvido pode ser positiva, principalmente pela contribuição ao bem-estar próprio e o de suas famílias (ALVES-MAZZOTTI, 1994; LIBÓRIO, 2009; MANTOVANI, 2012) e por uma crença de que através dele, elas estão adquirindo experiências que poderão lhes preparar melhor para futuros empregos, com melhores salários.

De um ponto de vista subjetivo, podemos observar na fala das crianças e adolescentes trabalhadores um forte senso de dignidade relacionado ao sentimento de autossuficiência, autoestima positiva, responsabilidade e contribuição na renda familiar obtidos por meio do trabalho realizado (FEITOSA; DIMENSTEIN, 2004). O trabalho infantil é um fenômeno multivariado, multidimensional e complexo que convida todos os envolvidos (gestores, professores, empregadores, comunidade, famílias, crianças e adolescentes) a olharem para os detalhes das experiências que o rodeia e a imaginarem as múltiplas perspectivas para lidar com suas complexidades, o que, por sua vez, irá levá-los a novos caminhos de ação.

Nessa direção, ao situar o trabalho a partir do ponto de vista de adultos com histórico de trabalho infantil no campo de estudos da resiliência, surgem debates polissêmicos. Quando considerado como um indicador de risco ao desenvolvimento é visto como atividade ligada aos prejuízos sobre a saúde e a escolarização. Em contrapartida, quando se analisa as subjetividades a ele ligadas, a renda, as possibilidades de aquisição de novas habilidades, as relações interpessoais estabelecidas, o sentimento de pertencimento, as aquisições materiais, entre outras, o olhar pode ser revestido de positividade, especialmente quando o trabalho traz reverberações no desenvolvimento da autoestima, responsabilidade, criatividade e socialização aos trabalhadores (LIBÓRIO, UNGAR, 2010; MANTOVANI, 2012; PESSOA, LIBÓRIO, VIOTTO FILHO, 2015).

A partir dessas considerações, tomamos como objetivo central nessa apresentação compreender o trabalho infantil em suas interlocuções com os estudos em resiliência a partir da fala de adultos com históricos de trabalho infantil estudantes em turmas de Educação de Jovens e Adultos (EJA) de Presidente Prudente/SP.

\section{METODOLOGIA}

Esse estudo está ancorado em uma abordagem qualitativa de pesquisa, pois toma os pressupostos trazidos por Ungar (2005a) e Ungar e Liebenberg (2013) para os estudos que se propõem a investigar processos de resiliência.

Buscamos delinear uma metodologia que nos ajudasse na compreensão dos aspectos subjetivos da experiência de adultos com o trabalho infantil, considerando o trabalho uma construção culturalmente embasada. Para isso, foi utilizado um questionário, embasado nos estudos de Ungar e Liebenberg (2013), e um roteiro de entrevista semiestruturado, elaborado a partir dos estudos de Mantovani (2012) e Santos (2011), para a coleta dos dados. As análises foram feitas a partir dos pressupostos da análise de conteúdo de Bardin (1977).

Participaram da pesquisa 131 adultos, entre 24 e 65 anos, de ambos os sexos $(53,4 \%$ mulheres), estudantes em duas escolas públicas estaduais de Presidente Prudente/SP que atendem na modalidade de ensino Educação de Jovens e Adultos (EJA). Tais instituições estão sob responsabilidade da Diretoria Regional de Ensino do município, tem características próprias e organização particular: a Escola A oferece aulas na EJA de forma flexível, com horários variados e nos três períodos do dia; já a Escola B tem aulas na EJA apenas no período noturno, em turmas fixas e organizadas anualmente.

Todos os participantes leram e assinaram o Termo de Consentimento Livre e Esclarecimento apresentado e tiveram suas identidades mantidas em sigilo. Ressalta-se, também, que a pesquisa teve a aprovação do Comitê de Ética em Pesquisa da Faculdade de Ciências e Tecnologia (FCT/UNESP) para sua execução sob o número de protocolo 27080814.6.0000.5402. 


\section{RESULTADOS}

A partir dos dados sociodemográficos constatou-se que a amostra foi composta por $53,4 \%$ de mulheres e 46,6\% de homens, residentes em Presidente Prudente e cidades da região, como Pirapozinho, Martinópolis, Alvares Machado, Caiabu, Narandiba e Alfredo Marcondes.

O rendimento mensal familiar dos participantes não obteve valores baixos, sendo significativa a porcentagem de sujeitos que tem uma renda mensal superior a três salários mínimos (25,9\%). No entanto, também é necessário considerar aqueles participantes que obtém uma renda mensal de até um salário mínimo $(15,3 \%)$ e aquele que não possui renda atualmente $(0,8 \%)$.

Com relação às implicações sobre a escolaridade dos participantes e seu envolvimento em situações de trabalho infantil solicitamos que indicassem o ano em que pararam de estudar, a série escolar e o(s) motivo(s) pelo(s) qual(is) tiveram que deixar a escola durante sua infância e/ou adolescência, bem como caracterizassem suas atividades de trabalho quanto ao local, tipo e frequência da atividade realizada, a decisão pelo trabalho e as formas de pagamento.

Os participantes deixaram de estudar entre os anos 1971 e 2000, no Ensino Fundamental II, que atualmente corresponde do 60 ano 9o ano escolar, por motivos que contemplam a inserção em trabalho $(56,5 \%)$, a falta de interesse pela escola $(16,8 \%)$, morar na área rural $(16 \%)$, morar longe da escola $(14,5 \%)$, a mudança de bairro/escola/cidade $(11,5 \%)$, a falta de dinheiro $(7,6 \%)$ e a qualidade da escola $(0,8 \%)$.

Sobre o envolvimento em situações de trabalho, o setor rural correspondeu a $35,1 \%$ dos participantes, seguidos daqueles envolvidos em trabalho no setor informal urbano (construção civil, loja, músico, carroceiro, restaurante, mercearia, fábrica, feira, circo, frigorífico, serralheria, tornearia, borracharia, empresa de ônibus, mecânica, panificadora, quadra de tênis, dentre outros), 29,8\%, e no trabalho doméstico, $26,7 \%$.

As atividades eram realizadas de 5 a 7 dias na semana (71,8\%) e a decisão pelo trabalho partiu, em sua maioria, pelo próprio participante (51,1\%), podendo contar com a decisão da mãe em $22,9 \%$ dos casos e do pai em $16,8 \%$. Quanto ao pagamento pela atividade realizada, $51,1 \%$ dos participantes relataram receber algum tipo de pagamento, enquanto $24,4 \%$ disseram não receber e $1,5 \%$ receber algum tipo de pagamento apenas de vezes em quando.

\section{DISCUSSÃO}

De acordo com os dados apresentados percebemos que o trabalho infantil persistiu ao longo do tempo, apesar da legislação disponível que prevê sua erradicação em todas as suas formas. Ao darmos voz a adultos com históricos de trabalho infantil, atualmente estudantes em turmas de EJA, percebemos que essa inserção em trabalho ocorreu, principalmente, em três modalidades (doméstico, rural e informal urbano), com destaque para o trabalho no setor rural, que, de acordo com a literatura da área, é a modalidade que mais emprega mão de obra infantil no mundo (STROPOLAS, 2012).

Por essa perspectiva subjetiva de análise, que considera os aspectos subjetivos, culturais, políticos, econômicos e emocionais dos próprios envolvidos em trabalho, as condições em que essas atividades se dão e os recursos disponíveis aos trabalhadores, percebemos que se torna necessário considerar, também, os aspectos positivos que coexistem com os fatores negativos, advindos de situações de trabalho infantil.

As reflexões de Carvalho (2009), Ferraz e Gomes (2012) e Sartori e Garcia (2012) perpassam essas discussões e destacam que o trabalho precoce oferece ganhos reduzidos e/ou inexistentes à educação e nega o direito a uma formação educacional e profissional que possibilite melhores condições de inserção futura. Segundo os autores, o tempo gasto com o trabalho diminui as possibilidades de estudo e aproveitamento nas atividades escolares, fatores esses que não serão recuperados facilmente e tendem a se propagar intergeracionalmente, implicando na 
baixa qualificação profissional futura e na maior probabilidade de recebimento de salários menores.

Porém, podemos perceber que o impacto do trabalho infantil sobre a pessoa adulta pode ser de diferentes dimensões e níveis, não devendo contemplar ações universalistas, "que podem ser redutoras dos sentidos atribuídos ao fenômeno, pois aspectos positivos podem estar coexistindo paralelamente a aspectos mais negativos nas experiências laborais de algumas crianças, enquanto para outras prevalecem apenas os fatores negativos" (LIBÓRIO, 2009, p. 43).

Sendo assim, além de considerar os aspectos negativos sobre a vida de adultos trabalhadores infantis, tanto em suas casas, como em fazendas e no setor informal urbano, é preciso analisar o tipo de atividade realizada e a frequência em que ela acontece; a existência ou não de algum tipo de pagamento pela atividade; a presença ou ausência de um adulto para supervisionar a atividade, no sentido de apoio, ensino e formação profissional; o local em que ocorre e os sentimentos advindos pela atividade, expressos pelo próprio trabalhador. Além disso, torna-se necessário observar os recursos disponíveis ao trabalhador que implicam diretamente no desenvolvimento de processos de resiliência (UNGAR, 2008).

\section{CONCLUSÃO}

Percebemos, nesse estudo, que para compreender o trabalho infantil em suas interlocuções com os estudos em resiliência é preciso dar voz aos próprios trabalhadores. Nesse sentido, ao situar o ponto de vista de adultos com histórico de trabalho infantil no campo de estudos da resiliência surgem debates polissêmicos.

Quando considerado como um indicador de risco ao desenvolvimento o trabalho é visto como atividade ligada aos prejuízos sobre a saúde e a escolarização. Em contrapartida, quando se analisam as subjetividades a ele ligas, os ganhos econômicos, as possibilidades de aquisição de novas habilidades, as relações interpessoais estabelecidas, o sentimento de pertencimento, as aquisições materiais, entre outra, o olhar pode ser revestido de positividade, especialmente quando traz reverberações no desenvolvimento da autoestima, da responsabilidade, da criatividade e da socialização dos trabalhadores.

\section{REFERÊNCIAS}

ALBERTO, M. F. P; NUNES, T. S.; CAVALCANTE, C. P.; SANTOS, D. P. O Trabalho Infantil Doméstico em João Pessoa - PB: um diagnóstico rápido à luz das piores formas de trabalho infantil. João Pessoa: OIT, 2005.

ALVES-MAZZOTTI, A. J. Do trabalho à rua: uma análise das representações sociais produzidas por meninos trabalhadores e meninos de rua. Coletivo de Autores. Rio de Janeiro: Diadorim UFRJ/CFCH, 1994.

BARDIN, L. Análise de Conteúdo. Lisboa: Edições 70 Lda, 1977.

BRASIL. Constituição da República Federativa do Brasil: promulgada em 05 de outubro de 1988. Organização do texto por Juarez de Oliveira. 4. ed. São Paulo: Saraiva, 1990.

CARVALHO, I. M. M. O trabalho infantil no Brasil Contemporâneo. Caderno CRH: Salvador, v. 21, n. 54, p.551-569, set/dez 2008. https://doi.org/10.1590/S0103-49792008000300010

FEITOSA, I. C. do N.; DIMENSTEIN, M. Escola, Família e Trabalho Infantil: Subjetividade e Práticas Disciplinares. Interação em Psicologia: Curitiba, v. 8, n. 2, p. 287-296, 2004. 
FERRAZ, L.; GOMES, M. H. A. Uma existência precarizada: o cuidado da prole no trabalho de catação de material reciclável. Revista Sociedade e Estado: Brasília, v. 27, n. 3, p. 652-662, set/dez 2012. https://doi.org/10.1590/S0102-69922012000300011

LIBÓRIO, R. M. C. Crianças e Adolescentes em situação de risco: dimensões do trabalho infantil nos municípios de Presidente Prudente, Belo Horizonte e Porto Alegre. Relatório de pesquisa não publicado (enviado ao CNPq), 2009.

LIBÓRIO, R. M. C.; UNGAR, M. Children Labour as a Risky Pathways to Resilience: children growth in contexts of poor resources. Psicologia Reflexão e Crítica: Porto Alegre, v. 23, n. 2, p. 232-242, 2010.

MANTOVANI, A. M. Trabalho Infantil e desenvolvimento na perspectiva de profissionais da educação e famílias. Dissertação (Mestrado em Educação) - Faculdade de Ciências e Tecnologia, Universidade Estadual Paulista, Presidente Prudente, 2012.

NUNES, I. B. O trabalho infantil na Revolução Industrial inglesa: uma contribuição ao trabalho docente na sétima série, 2009. Disponível em http://www.diaadiaeducacao.pr.gov.br/portals/pde/arquivos/1397-8.pdf, acesso em maio de 2015.

SANTOS, D. P. dos. As implicações psicossociais do trabalho precoce em adultos. Dissertação (Mestrado em Psicologia Social) - Programa de Pós-graduação em Psicologia Social, Universidade Federal da Paraíba, João Pessoa, 2011.

SARTORI, E.; GARCIA, C. H. M. Políticas compensatórias versus emancipatórias: desafios para implementação de programas de geração de renda às famílias em situação de risco. RAP: Rio de Janeiro, v. 46, n. 2, p. 425-452, mar/abr 2012.

STROPASOLAS, V. L. Trabalho Infantil no campo: do problema social ao objeto sociológico. Revista Latino-Americana de Estudos do Trabalho, v. 17, n. 27, 2012, p. 249-286.

UNGAR, M. Introduction: Resilience across cultures and contexts. In: UNGAR, M. (ed.). Handbook for working with children and youth: Pathways to resilience across cultures and contexts, Thousand Oaks: Sage, p. xv-xxxix, 2005a. https://doi.org/10.1142/97898127032790001 . Resilience across culture. British Journal of Social Work, v. 38, n. 2, p. 218-235, 2008.

UNGAR, M.; LIEBENBERG, L. The Resilience Research Centre Adult Resilience Measure (RRC-ARM) - User's manual: Research, 2013. 